\title{
Anterior sacral meningocele mimicking ovarian cyst: a case report.
}

\author{
Ahmet Veysel Polat ${ }^{1}$, Umit Belet ${ }^{1}$, Ramazan Aydin ${ }^{1}$, Seckin Katranci ${ }^{2}$ \\ ${ }^{1}$ Department of Radiology, University of Ondokuz Mayis, Samsun, Turkey \\ ${ }^{2}$ Buyuk Anadolu Hospital, Samsun, Turkey
}

\begin{abstract}
Anterior sacral meningocele (ASM) is a rare congenital anomaly, characterized by herniation through a defect in the anterior aspect of the sacrum. We reported a case of ASM associated with neurofibromatosis, which was mimicking a complex ovarian cyst in ultrasonography. ASM can easily be misdiagnosed as an ovarian cyst and needle aspiration can cause intracranial hypotension syndrome or even death.
\end{abstract}

Keywords: neurofibromatosis type 1, anterior sacral meningocele, ultrasonography, magnetic resonance imaging

\section{Introduction}

Anterior sacral meningocele (ASM) is a rare congenital anomaly, characterized by herniation through a defect in the anterior aspect of the sacrum [1]. Since the first ASM case description published in the 1837, fewer than 300 cases have been reported so far [2]. ASM mostly occurs sporadically, but it may be seen in conditions with dural ectasia such as neurofibromatosis type 1 (NF-1) and Marfan's syndrome [3]. ASM associated with NF is a rare clinical entity and may present as a pelvic cyst. If not evaluated thoroughly, intervention to this cyst may result in severe complications. We report a case of ASM associated with NF incidentally identified by ultrasonography (US) and the magnetic resonance imaging (MRI) findings of ASM and particular advantages of this imaging method in diagnosis of ASM are discussed.

Received 17.08.2012 Accepted 14.10.2012

Med Ultrason

2013, Vol. 15, No 1, 67-70

Corresponding author: Dr. Ahmet Veysel Polat

Ondokuz Mayis University Faculty of

Medicine Department of Radiology

55139 Samsun, Turkey

Tel: $90.362 .3121919 / 2068$

Fax: 90.362.4576041

E-mail: veyselp@hotmail.com

\section{Case Report}

An ASM was incidentally identified during abdominopelvic ultrasonography for nonspecific right upper quadrant pain examination in a 16-year-old girl with NF1 , diagnosed 8 years earlier. She had no complaints attributable to ASM. She had characteristic skin lesions, café au lait macules, distributed over the different sites of body. Routine blood chemistry results were within normal limits. Cranial and orbital computed tomography (CT) examinations were normal. Cranial MRI demonstrated hamartomatous lesions.

The pelvic sonographic examination revealed a multiloculated cystic mass with $6 \times 5 \times 5 \mathrm{~cm}$ dimensions located in presacral spaces, posterior to uterus and bladder (fig 1). Although it resembled an ovarian cyst, careful examination revealed that it was connected to the dural sac. A diagnosis of ASM was suggested, and plain radiographs, CT and MRI were performed for confirmation.

There were enlargement and deformation of intervertebral foramina on plain radiographs of pelvis (fig 2). Pelvic $\mathrm{CT}$ confirmed the presence of a large, multiloculated, smooth surface fluid filled-mass in the precasral space with associated bony defects (fig 3). Contrast enhanced MRI demonstrated large, multiloculated intrasacral and presacral cysts communicating via three separate broad 


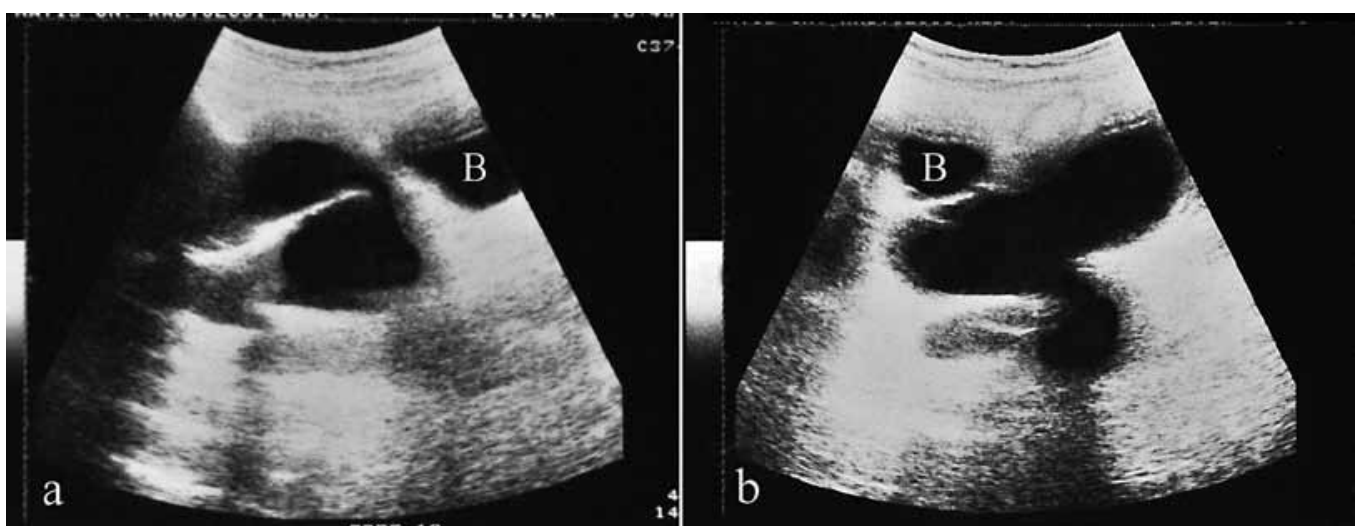

Fig 1. Sagittal (a), axial (b) US scan shows cystic structures with no solid component at presacral region behind bladder (B:bladder)

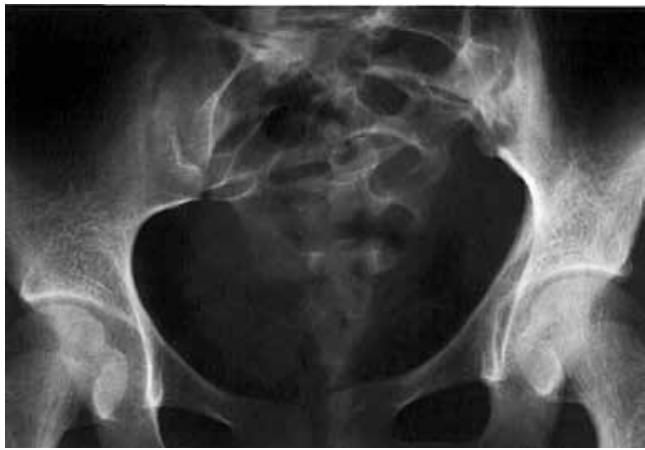

Fig 2. Plain radiography of pelvis depicts widened and deformed sacral neural foramina

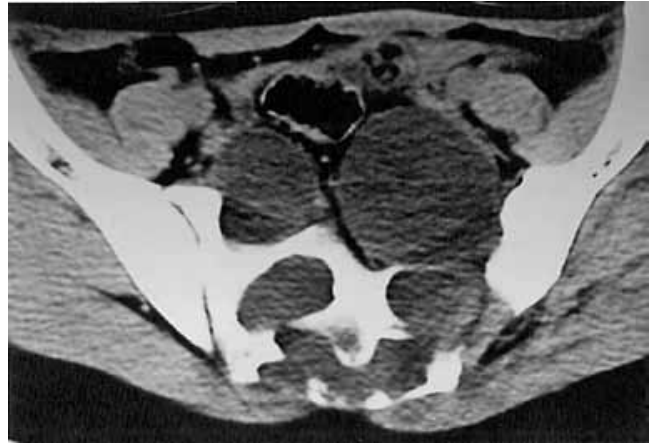

Fig 3. Axial unenhanced CT shows cystic lesions with distinct borders related with spinal canal. Lesions had caused widening and destruction at sacrum and neural foramina.
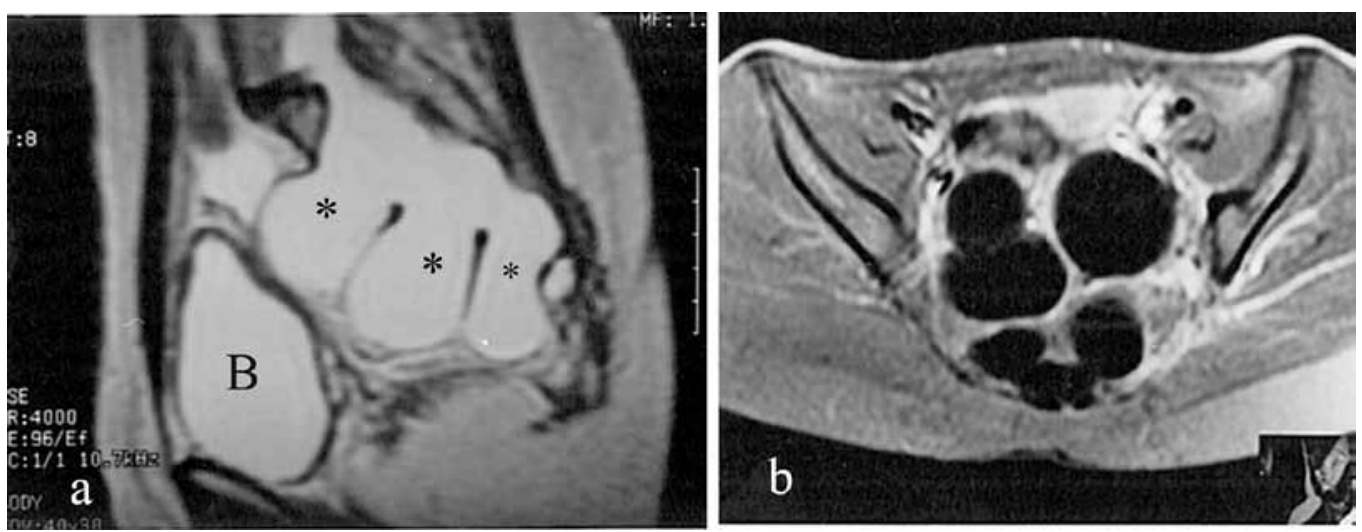

Fig 4. T2 weighted sagittal MR image depicts wide sacral sac extending into pelvis through 3 separate broad necks, b. T1 weighted fat suppressed contrast enhanced axial image displays no contrast enhancement (B: bladder,* ASM). 
necks and extending through the sacral neural foramina. The lesion was hypointense on T1-weighted images, hyperintense T2-weighted images and no contrast enhancement was detected. A connection between this lesion and dural sac was clearly observed on sagittal MRI images. There were neither solid component nor neural elements (fig 4). The final diagnosis was ASM. The patient was informed about surgical management and non-operative follow-up. Since the patient had no symptoms and neurological deficits, surgery was not performed.

\section{Discussion}

ASM occurs as a result of a rare congenital defect characterized by focal erosion or hypogenesis of segments of the sacrum and coccyx with herniation of a cerebrospinal fluid (CSF)-filled meningeal sac through the defect into the pelvis. It accounts for about $5 \%$ of retrorectal masses and is usually diagnosed in the second and third decades of life. Neural elements may occasionally be found in the sac. ASM is either asymptomatic or manifested by nonspecific symptoms as a result of pressure on the viscera such as constipation, urinary problems, dysmenorrhea, or pain in the lower back or pelvis [4,5]. Furthermore pressure may be exerted on nerve roots, resulting in sciatica, diminished rectal and detrusor tone, or numbness and paresthesia in the lower sacral dermatomes. In addition, fluid shifts between the sac and the spinal subarachnoid space can cause intermittent low or high-pressure headache, nausea and vomiting related to changes in body position [2]. Very few cases have been asymptomatic, as in our case [6].

$\mathrm{NF}$, autosomal dominant disease with variable penetrance, is characterized by well-recognized skin lesions, neural tumors, and skeletal abnormalities, most commonly scoliosis and kyphosis. High frequency of bone abnormalities has been reported in patients with NF1 [7]. Meningocele is a less frequently associated lesion in patients with NF1. These meningoceles, occurring through bony defects, are primarily anterolateral in relation to the spine in contradistinction to the posterior meningoceles of spinal dysraphism. ASM in patients with NF was first described by Oren in 1977 [8]. Several ASM cases were reported in literature, but there are only five reported cases of ASM associated with NF [8-11].

ASM has been reported to be misdiagnosed as ovarian cyst on many occasions [4,12]. A detailed clinical examination and radiological investigations should be able to differentiate ASM from other causes of cystic presacral masses which include ovarian cyst, rectal duplication cyst, neuroectodermal cyst, perineural cyst and sacrococcygeal teratoma [5].
Diagnosis of meningocele can frequently be made by rectal examination. The other diagnostic studies include plain radiograph, US, myelography, barium enema, CT, excretory urography, and MRI. Imaging studies show a deficient sacrum and a variably sized cyst extending into the pelvis through an enlarged sacral foramen. In order to clarify the diagnosis, continuity of the cyst with the thecal sac must be demonstrated $[6,13]$.

In plain radiographs, the curved appearance of the residual sacrum, scalloped beneath the defect with an appearance of scimitar sacrum, is considered as an almost pathognomonic finding, and is present in $50 \%$ of cases [14].

US, as a screening tool, often reveals the presence of the intra-abdominal cystic abnormality. However, before any manipulation such as needle aspiration is performed for the suspected ovarian cyst in Douglas pouch, a plain radiograph must be obtained to look for sacral abnormalities for the patients diagnosed with NF [14]. ASM is seen as a fluid-filled cyst via US and careful examination should be made to avoid misdiagnosis as an ovarian cyst or filled urinary bladder [4]. US may also have a role in the follow up of the size changes in cystic masses.

$\mathrm{CT}$ is useful to display bony anomalies and erosions and CSF density within the cyst [6]. Intrathecal contrast enhanced CT scanning is the diagnostic procedure of choice, since it not only demonstrates the communication between the meningocele and the subarachnoid space but also usually demonstrates associated tumors if they cause filling defects [13]. However, this method is invasive and has the disadvantage of ionizing radiation.

MRI is a safe, rapid, and noninvasive imaging method with multiplanar imaging capability, and it can also show any associated small tumors. Accurate information of the shape, size, anatomical relations to the surrounding organs, and internal characteristic of the cystic mass may be obtained with routine MRI without introduction of contrast material into the spinal canal [13]. MRI is capable of showing characteristic CSF intensity within the cyst, without the need for intrathecal contrast material. MRI can provide nearly all the information derived from CTmyelography, an exception may be demonstration of subarachnoid communication in small-necked lesion [14].

Since ASM does not regress, symptomatic ASM requires surgical intervention for the patient's comfort and safety. Opinions on management diverge according to different authors in cases of asymptomatic ASM. Some authors recommend surgery in all cases to avoid infectious complications. Others recommend conservative management, suggesting surgical intervention only in the case of symptoms or increase in size of the lesion due to increased hydrostatic pressure [2]. 
In conclusion, ASM can easily be misdiagnosed as an ovarian cyst and needle aspiration can cause intracranial hypotension syndrome or even death. The diagnosis of ASM should always be kept in mind in cases with pelvic cysts having prior diagnosis of NF. Pelvic US may be a screening tool for ASM in patients with NF. MR imaging is a safe and effective method of demonstrating all the characteristic of ASMs.

\section{References}

1. Villarejo F, Scavone C, Blazquez MG, et al. Anterior sacral meningocele: review of the literature. Surg Neurol 1983; 19: $57-71$

2. Manson F, Comalli-Dillon K, Moriaux A. Anterior sacral meningocele: management in gynecological practice. Ultrasound Obstet Gynecol 2007; 30: 893-896.

3. Schneider MB, Dittmar S, Boxer RA. Anterior sacral meningocele presenting as a pelvic/abdominal mass in a patient with Marfan syndrome. J Adolesc Health 1993; 14: 325-328.

4. Erdogmus B, Yazici B, Ozdere BA, Safak AA. Anterior sacral meningocele simulating ovarian cyst. J Clin Ultrasound 2006; 34: 244-246.

5. Mohta A, Das S, Jindal R. Anterior sacral meningocele presenting as constipation. J Pediatr Neurosci 2011; 6: 40-43.
6. North RB, Kidd DH, Wang H. Occult, bilateral anterior sacral and intrasacral meningeal and perineurial cysts: case report and review of the literature. Neurosurgery 1990; 27: 981-986.

7. Nakasu Y, Minouchi K, Hatsuda N, Nakasu S, Handa J. Thoracic meningocele in neurofibromatosis: CT and MR findings. J Comput Assist Tomogr 1991; 15: 1062-1064.

8. Oren M, Lorber B, Lee SH, Truex RC Jr, Gennaro AR. Anterior sacral meningocele: report of five cases and review of the literature. Dis Colon Rectum 1977; 20: 492-505.

9. Mellion BT, George RE, Fischer DK, Lidsky MD, Baskin DS. Anterior sacral meningocele and tuberculous spondylitis of the sacrum in a patient with neurofibromatosis. Case report and review of the literature. Neurofibromatosis 1989; 2: 299-308.

10. Aygun C, Tekin MI, Tarhan C, Ozdemir H, Peskircioglu L, Ozkardes H. Neurofibroma of the bladder wall in von Recklinghausen's disease. Int J Urol 2001; 8: 249-253.

11. Green WJ, Green AE Jr. Large pelvic mass in a patient with neurofibromatosis. Invest Radiol 1988; 23: 772-774.

12. Ficicioglu C, Baykal C, Arioglu P, Delier H, Mercan A, Ozcan N. Anterior meningocele misdiagnosed as an ovarian cyst. Gynecol Obstet Invest 2008; 65: 21-23.

13. Lee KS, Gower DJ, McWhorter JM, Albertson DA. The role of MR imaging in the diagnosis and treatment of anterior sacral meningocele. Report of two cases. J Neurosurg 1988; 69: 628-631.

14. Martin B, Boyer de Latour F. MR imaging of anterior sacral meningocele. J Comput Assist Tomogr 1988; 12: 166-167. 